\title{
Ein dezentraler Regelalgorithmus für ein automatisches Koexistenzmanagement
}

\author{
Darina Schulze, Ulrich Jumar \\ ifak e.V. Magdeburg \\ Werner-Heisenberg-Straße 1, 39106 Magdeburg \\ darina.schulze@ifak.eu \\ ulrich.jumar@ifak.eu
}

Zusammenfassung. Das Konzept Industrie 4.0 sieht steigende Flexibilität und Mobilität im Produktionsprozess vor. Im industriellen Funkkommunikationsbereich werden zunehmend höhere Anforderungen an das Zeit- und Fehlerverhalten einer Nachrichtenübertragung gestellt. Dabei ist zu beachten, dass sich die Kommunikationsanforderungen im Laufe der Zeit ändern können und gegebenenfalls neue Anwendungen wichtiger als bereits vorhandene werden. In Folge dieser gestellten Kommunikationsanforderungen kommen unterschiedliche Funkkommunikationssysteme $(W C S)$ zum Einsatz. Diese $W C S$ können auf der Basis unterschiedlicher Technologien (WiFi, Bluetooth oder DECT) implementiert sein. Wenn mehrere WCSs auf dem gleichen Frequenzband, zur selben Zeit und im selben Raum übertragen, können sie sich gegenseitig beeinflussen, bzw. interferieren. Die WCSs sollten dabei koexistieren. Der Begriff der Koexistenz wurde in [IE14] mit der folgenden Definition belegt: "Koexistenz ist ein Zustand, in dem alle Funkkommunikationslösungen einer Anlage, die ein gemeinsames Medium nutzen, alle Kommunikationsanforderungen ihrer Anwendung erfüllen". Maßnahmen, die zum Erreichen bzw. Beibehalten der Koexistenz beitragen, werden Koexistenzmanagement genannt. Aktuell wird in der industriellen Funkkommunikation verbreitet das manuelle Koexistenzmanagement angewendet. Um das zeitliche Verhalten, hinsichtlich der Reaktion auf eine Störung, weiter zu optimieren, befassen sich wesentliche Forschungsschwerpunkte mit dem automatischen Koexistenzmanagement in der industriellen Automation. In diesem Beitrag wird ein dezentraler Regelalgorithmus für ein dezentrales Koexistenzmanagement vorgestellt und an einem Hardware in the Loop Aufbau validiert. Das besondere hieran ist, dass der Ansatz anwendungsbasiert und technologieunabhängig ist. Ergebnisse zur Modellierung der Regelstrecke, welche im Zusammenhang mit der drahtlosen Kommunikation als Koexistenzstrecke bezeichnet wird, wurden in [Sc17] und [SZJ18] gezeigt. Hierbei wird das zeitliche Übertragungsverhalten einer Nachricht, im ungestörten und gestörten Fall, modelliert und bewertet. Als Regelgröße dafür wird die Übertragungszeit (engl. Transmission time) daher nachgebildet. Diese entspricht in der Realität die Messgröße. Als Methodik zur Modellierung wird die Petrinetznotation in der max - plus Algebra gewählt. Diese Methodik hat den Vorteil, dass das Streckenverhalten als lineares Systemverhalten nachgebildet werden kann. Der dezentrale Regelalgorithmus bezieht sich auf die 
Methodik in der max - plus Algebra. Hierbei wird ein modellprädiktives Verfahren (engl. Model predictive control $M P C$ ) angewendet. Dieses beinhaltet die Definition einer Zielfunktion, welche eine Anzahl von Nebenbedingungen unterliegt. Um den Regelalgorithmus zu validieren, wird ein industrienaher Hardware in the Loop Aufbau gewählt. Hieran werden einzelne Testszenarien mit mehreren WCSs definiert, wobei sich die $W C S s$ gegenseitig stören. Störung heißt in diesem Falle, dass das $W C S$ den Koexistensbereich verlässt. Der Regler, soll das jeweilige $W C S$ in den Koexistenzbereich zurückführen.

\section{Einführung}

Die aktuellen Konzepte von Industrie 4.0, sowie von $5 G$ und die damit verbundene Digitalisierung spielen in der Funkkommunikation eine wesentliche Rolle. Damit verbunden sind die zunehmenden Anforderungen an das Zeit- und Fehlerverhalten der Nachrichtenübertragungen von Funkkommunikationssystemen (WCSs). Wenn mehrere WCSs den selben Raum-, Frequenz- und Zeitbereich nutzen, können diese Nachrichtenübertragungen beeinflusst werden. Unter Beeinflussung wird verstanden, wenn die Nachrichten zeitlich verzögert oder zerstört werden. Die WCSs können somit untereinander Interferieren, sodass die Koexistenz verloren geht. Der Begriff der Koexistenz wurde in [IE14] mit der folgenden Definition belegt: "Koexistenz ist ein Zustand, in dem alle Funkkommunikationslösungen einer Anlage, die ein gemeinsames Medium nutzen, alle Kommunikationsanforderungen ihrer Anwendung erfüllen". Das Koexistenzmanagement versucht unter speziellen Maßnahmen hier in den Prozess einzugreifen, um so die Koexistenz wieder herzustellen. In der Literatur gibt es zahlreiche Konzepte für die Bestimmung eines, manuellen oder automatischen, Koexistenzmanagements. In der vorliegenden Forschungsarbeit wird sich auf ein automatisches Koexistenzmanagement konzentriert. Das automatische Koexistenzmanagement teilt sich in ein kooperatives und ein nicht kooperatives Management auf. Auf das nicht kooperative Management wird hier nicht weiter eingegangen. Der Leser kann sich dazu in [AMW12], [ASM11], [GTS08], [Ha05], [YA09] informieren. Ein automatisches und kooperatives Koexistenzmanagement bedeutet, dass die WCSs Informationen untereinander austauschen und entsprechend reagieren können. Darin unterschieden wird zwischen einem zentralen und einem dezentralen Ansatz. Ein zentrales Management hat eine zentrale Instanz, dies kann z. B. eine zentrale Koordinierungseinheit (englisch Central Coordination Point) sein. Aktuell wird es in der Literatur als eine Einheit beschrieben, welche den Status der Nachrichtenübertragungen aller WCSs einsammelt. In [KR15] wird ein Konzept solcher Instanz beschrieben. Jedoch fehlt zu diesem Konzept ein entsprechender Algorithmus, der für künftige Untersuchungen aussteht. Eine weitere Methodik für ein Koexistenzmanagement ist der dezentral kooperative Ansatz. In [Su12] gibt es dazu eine Beschreibung zum Einsatz eines Secondary User Networks mit Primary User Constraints. Die Autoren führen hierzu einen Optimierungsparameter, Quality of Coexistence (QoC), ein. Diese Methodik ist 
jedoch auf die WiFi -Technologie spezialisiert und ist daher nicht geeignet für ein technologieunabhängiges automatisches Koexistenzmanagement.

Viele der erwähnten Methoden, welche in der Literatur zum Koexistenzmanagement bestehen, sind abhängig von der jeweiligen Funktechnologie. Im Gegensatz zu dem aktuellen Literaturüberblick bezieht sich der vorliegende Beitrag auf die Untersuchung eines anwendungsbasierten und technologieunabhängigen automatischen Koexistenzmanagements. Solch einen Ansatz gibt es aktuell nicht. Um diese Eigenschaft eines Koexistenzmanagementsystems zu erreichen sollen Methoden aus der Regelungstechnik untersucht werden. In [SR16] kann sich der Leser einen ersten Überblick zu dem Konzept machen.

In dem vorliegenden Beitrag wollen die Autoren einen dezentralen Ansatz für ein technologieunabhängiges automatisches Koexistenzmanagement zeigen. Die entsprechende Regelstrecke wurde in [Sc17] und [SRJ17], für den interferenzfreien und Interferenzfall, modelliert und untersucht. Dort wurde die Methodik der zeitbehafteten Petrinetzmodellierung in der max - plus Algebra angewendet. Im Bereich der Regelung wird in diesem Beitrag die modellprädiktive Regelung (englisch Model Predictive Control, MPC) in der max - plus -Algebra auf die Anwendung eingesetzt. Die Validierung der Regelung wird an einem Hardware in the Loop Aufbau durchgeführt.

Nomenklatur Die Menge $\mathbb{R}_{\max }=(\mathbb{R} \cup\{-\infty\} \cup\{\infty\})$ wird als max-plus Algebra mit zwei Operationen $q \oplus w=\max (q, w)$ und $q \otimes w=q+w, \forall q, w \in \mathbb{R}_{\max }$ beschrieben. Das neutrale Element mit Bezug auf $\oplus$ ist $\varepsilon \longrightarrow-\infty$, sodass $\varepsilon \oplus w=\max (\varepsilon, w)=w$. Das neutrale Element mit Bezug auf $\otimes$ ist $e:=0$, sodass $e \otimes w=0+w=w$. Weiterhin wird die Modellbeschreibung in Zustandsraumnotation für ereignisdiskrete Systeme mit $x(k+1)=f(x(k))$ genutzt.

\section{Der Betrachtungsraum als Regelkreis}

Für die Formulierung der Koexistenz in ein regelungstechnisches Problem, wird der Betrachtungsraum eingeführt. Abbildung 1 beschreibt dabei eine Erweiterung des Betrachtungsraum von [Sc17], wo der Betrachtungsraum als Regelstrecke beschrieben ist. In diesem Beitrag wird der Betrachtungsraum um die dezentrale Regelung erweitert. Jedes $W C S$ besitzt mindestens eine Quelle (englisch Source $S$ ) und mindestens ein Ziel (englisch Target $T$ ), welche als drahtlose Geräte (englisch Wireless Device $W D$ ) beschrieben sind. In Abbildung 1 bildet jedes $W D$ mit der gleichen Nummerierung ein $W C S$. Um die Eindeutigkeit der Nachrichtenübertragung festzustellen, werden logische Links $l$ und physikalische Links $p l$ eingeführt. Ein logischer Link beschreibt die Verbindung zwischen zwei logischen Endpunkten von einer automatisierungstechnischen Anwendung. Ein physikalischer Link beschreibt die Verbindung von Antenne zu Antenne. Bezüglich eines WCS beschreibt es den Funkkanal. Die Menge aller Funkkanäle beschreibt das Übertragungsmedium. Wenn ein WCS unabhängig von den anderen WCSs seine Nachrichten überträgt, kann es passieren, dass sich diese Funkkanäle überlagern. Dabei können Interferenzen entstehen. Für die Minimierung dieser Interferenzen wird eine dezentrale Regelung eingeführt. In Abbildung 


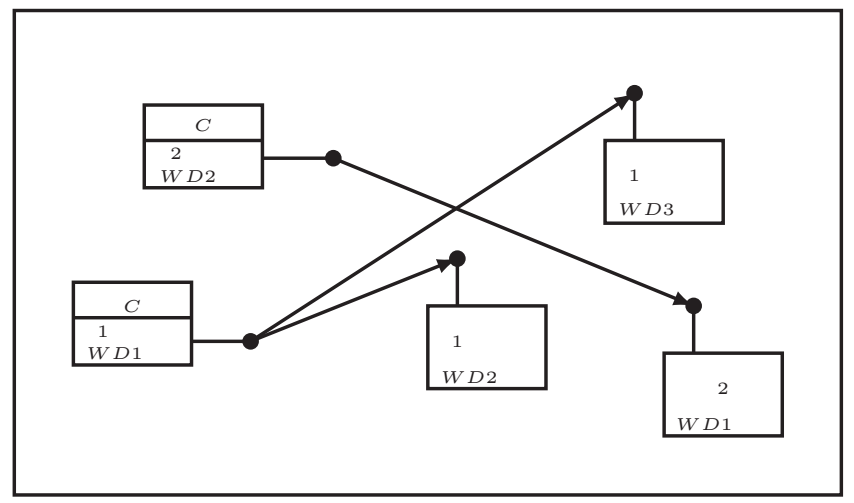

Abb. 1. Beschreibung des Betrachtungsraumes nach [SZ18a]

1 wird die Regelung mit $C$ (englisch Controller) beschrieben. Jedes $W C S$ hat, so viele $C$ wie $S$ vorhanden sind, vorausgesetzt die Nachrichtenübertragung ist unidirektional. Ist die Übertragung bidirektional, wird das $T$ zur $S$ und ist ebenso mit einem $C$ versehen. Eine weitere Eigenschaft des Betrachtungsraums liegt in der Beschreibung der Signale. Dazu zeigt Abbildung 2 einen gewöhnlichen Regelkreis auf, welcher Zugleich eine Analogie zur Thematik der Funkkommunikation liefern soll [SR16]. Die Regelstrecke, welche das Koexistenzverhalten beschreibt,

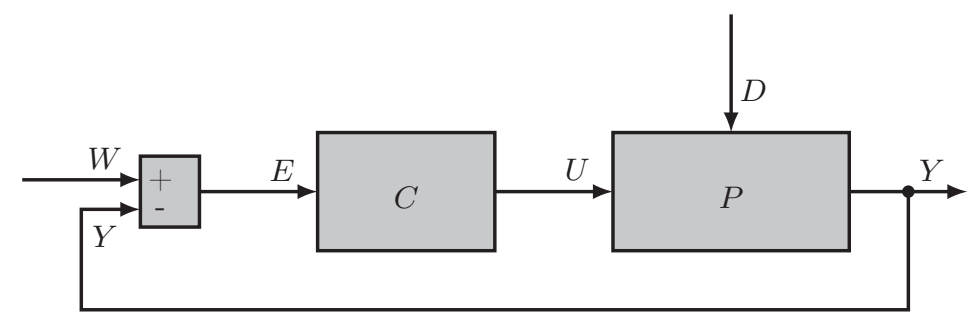

Abb. 2. Struktur eines Regelkreises nach [In13]

wird mit $P$ bezeichnet. Sie ist die Menge aller WCSs in der Fabrikhalle. Ein $W C S$ wird als Sub-plant $p^{(i)} \in P$ beschrieben, wobei $i \leq s$ und $s$ die Gesamtanzahl von $p^{(i)}$ ist. Ein $p^{(i)}$ besteht für die Funkkommunikation aus mindestens zwei $W D$, welche mit mindestens einem logischen und mindstens einem physikalischen Link verbunden sind, daher $p^{(i, l)}$. C beschreibt das Verhalten einer Regelung, zentral oder dezentral, für die Koexistenz. Aus regelungstechnischer Sicht bedeuten die Signale $W^{(i, l)}, Y^{(i, l)}, U^{(i, l)}, E^{(i, l)}$ und $D^{(i, l)}$ der Führungs-, der Mess- (Regel), der Stell-, der Fehler- und der Störparametervektor pro $p^{(i)}$, aus Kommunikationssicht wiederum die charakteristische Referenz-, der charakteristische Mess-, der Einfluss- für $U^{(i, l)}$, der Fehler- und für $D^{(i, l)}$ der aktive 
Umgebungsparametervektor. Im nachstehenden Kapitel wird eine Zusammenfassung zur Modellierung der Regelstrecke von [Sc17] und [SZJ18] gegeben, welches als Grundvoraussetzung für den Regelentwurf dient.

\section{Modellierung der Regelstrecke zur Koexistenz}

Die Modellierung der Regelstrecke soll eine zeitliche Entkopplung der Nachrichtenübertragung eines $W C S$ zu den anderen $W C S s$ darstellen. Das Ziel der Regelung soll es somit sein, eine zeitliche Verschiebung der Nachrichtenübertragung am WCS zu erzeugen. Als Stellgröße soll dabei der Sendezeitabstand (englisch Transfer Intervall) gewählt werden, Eingangsgröße $u$ der Regelstrecke. Die Regelgröße stellt die Übertragungszeit (englisch Transmission time) dar, Ausgangsgröße $y$ der Regelstrecke. Die zeitliche Beschreibung der Ein- und Ausgangsgröße soll hierbei als Ereignis der Nachrichtenübertragung aufgefasst werden. Dabei soll die Methodik der zeitbehafteten Petrinetze herangezogen werden. Die nachstehende Abbildung 3 zeigt die Grundstruktur der Regelstrecke auf. Die Notation des Petrinetzgraphen ist $G=(\theta, \lambda, \xi, \nu)$, wobei $\theta$ die Men-

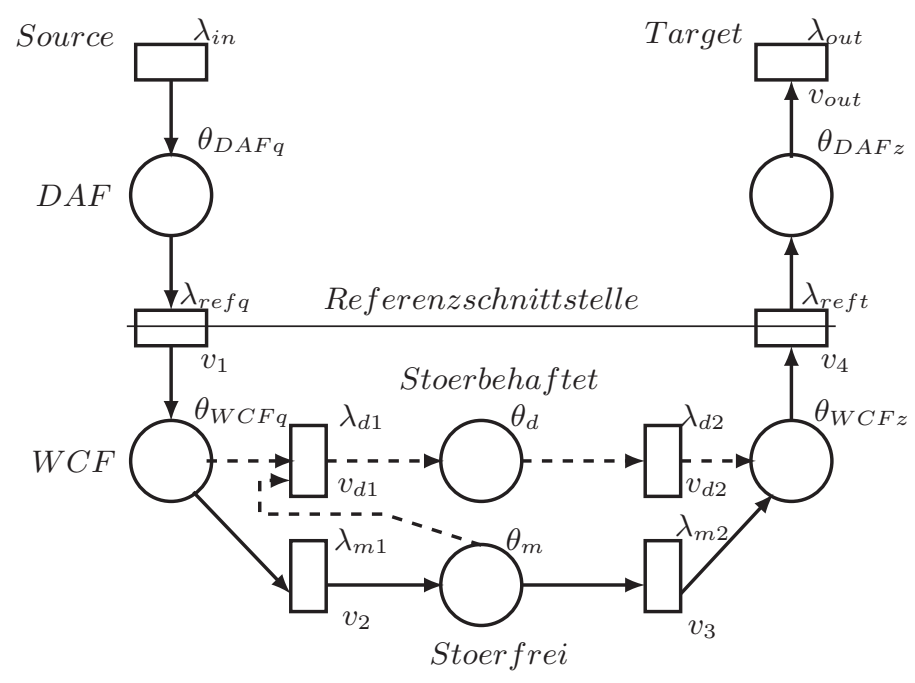

Abb. 3. Struktur der Regelstrecke $P$ für die Koexistenz nach [Sc17]

ge der Plätze, $\lambda$ die Menge der Transitionen, $\xi$ die Menge der Kanten und $\nu$ die Menge der Wichtungen sind. Die Funktionalität des $W D$ wird in Distributed Application Function $(D A F)$ durch $\theta_{D A F}$ und in Wireless Communication Function $(W C F)$ durch $\theta_{W C F}$ aufgeteilt. Die $S, D A F$ ist verantwortlich für die Generierung der Nachrichten und die $S W C F$ ist für beispielsweise den Medienzugriff verantwortlich. Die $T, W C F$ gibt eine Aussage, ob die Übertra- 
gung der Nachrichten erfolgreich gewesen ist. Für den Koexistenzmanagementprozess bedeutet dies, dass die $D A F$ die Regelgröße der Transmission time ausgibt und den Koexistenzzustand bewertet. Das Medium wird durch $\theta_{m}$ für den störfreien Zustand beschrieben und $\theta_{d}$ stellt des störbehafteten Zustand mehrerer WCSs dar. Für das Interferenzverhalten ist die Menge der Plätze wie folgt $\left.\theta_{D A F s}, \theta_{W C F s}, \theta_{m}, \theta_{d}, \theta_{D A F t}, \theta_{W C F t}\right\} \in \theta^{(i, l)}$. Die Transitionen $\lambda_{\text {refs }}$ und $\lambda_{\text {reft }}$ repräsentieren die Referenzschnittstelle in der $S$ und die Referenzschnittstelle in der T. $\lambda_{m 1}$ repräsentiert die Transition zur Nachrichtenübertragung von der $S$ auf das Medium und $\lambda_{m 2}$ repräsentiert die Transition zur Nachrichtenübertragung von dem Medium in das T. Die Transitionen $\lambda_{d 1}$ und $\lambda_{d 2}$ sind für den Interferenzfall bestimmt, wenn mehrere WCSs aktiv sind. Die Menge aller Transitionen lässt sich daher bestimmen zu $\left\{\lambda_{\text {refs }}, \lambda_{m 1}, \lambda_{m 2}, \lambda_{d 1}, \lambda_{d 2}, \lambda_{\text {reft }}\right\} \in \lambda^{(i, l)}$. Bei den Transitionen handelt es sich um zeitbehaftete Transitionen. Die Zeitverzögerungen an den Transitionen werden durch $\left\{v_{1}, v_{2}, v_{3}, v_{d 1}, v_{d 2}, v_{4}, v_{\text {out }}\right\} \in v$ beschrieben. Für die Modellierung von $P$ wir der Zustandsvektor $x^{(i, l)}$ pro $p^{(i, l)}$ eingeführt. Dieser beschreibt die Menge aller Zeitstempel, wann die jeweiligen Transitionen feuern. Für den Reglerenwurf soll in der Prädiktion der störfreie Pfad genutzt werden. Daraus ergibt sich in der Analogie zwischen der regelungstechnischen Notation und der Notation für die Petrinetze für $x^{(i, l)}=\left\{x_{1}, x_{2}, x_{3}, x_{4}, x_{c}\right\}$ mit $\lambda^{(i, l)}=\left\{\lambda_{\text {refs }}, \lambda_{m 1}, \lambda_{m 2}, \lambda_{\text {reft }}, \lambda_{\text {out }}\right\}$. Der Ausgang von $P$ ist $x_{4}$, welcher mit $x_{c}$ gleichzeitig die Eingangsgröße des Reglers ist. Das Zeitverhalten für eine Nachrichtenübertagung für den störfreien Zustand wird pro logischem Link für $p^{(i, l)}$ im Zustandsraum mit [SRJ17]

$$
p^{(i, l)}:\left\{\begin{array}{l}
x^{(i, l)}(k+1)=f\left(x^{(i, l)}(k), u^{(i, l)}(k+1)\right) \\
y^{(i, l)}(k)=h\left(x^{(i, l)}(k)\right)
\end{array}\right.
$$

beschrieben. Die Verzögerungen $v$ an den Transitionen haben eine nicht lineare Eigenschaft für das Systemverhalten zur Folge. Daher wird eine max - plus Transformation durchgeführt, um ein lineares Systemverhalten zu erhalten. Für den Regler wird die Annahme getroffen, dass $\theta_{D A F t}$ eine Nachricht hat, welche die Regelgröße beinhaltet. Dieser Aspekt ist eine Erweiterung zu dem Zustandsraummodel aus [Sc17] und [SRJ17]. Die allgemeine Beschreibung von $x^{(i, l)}(k)$ kann formuliert werden als

$$
\begin{aligned}
x^{(i, l)}(k+1) & =A_{0} x^{(i, l)}(k+1) \oplus A_{1} x^{(i, l)}(k) \oplus B u^{(i, l)}(k+1) \\
y^{(i, l)}(k) & =C x^{(i, l)}(k),
\end{aligned}
$$


mit

$$
\begin{aligned}
& A_{0}=\left(\begin{array}{ccccc}
\varepsilon & \varepsilon & \varepsilon & \varepsilon & \varepsilon \\
v_{2} & \varepsilon & \varepsilon & \varepsilon & \varepsilon \\
\varepsilon & v_{3} & \varepsilon & \varepsilon & \varepsilon \\
\varepsilon & \varepsilon & v_{4} & \varepsilon & \varepsilon \\
\varepsilon & \varepsilon & \varepsilon & \varepsilon & \varepsilon
\end{array}\right) \quad A_{1}=\left(\begin{array}{ccccc}
\varepsilon \varepsilon & \varepsilon & \varepsilon & \varepsilon \\
\varepsilon \varepsilon & \varepsilon & \varepsilon & \varepsilon \\
\varepsilon \varepsilon & \varepsilon & \varepsilon & \varepsilon \\
\varepsilon \varepsilon & \varepsilon & \varepsilon & \varepsilon \\
\varepsilon & \varepsilon & v_{\text {out }} & \varepsilon
\end{array}\right) \\
& B=\left(\begin{array}{c}
v_{1} \\
\varepsilon \\
\varepsilon \\
\varepsilon \\
\varepsilon
\end{array}\right) \quad C=\left(\begin{array}{ccc}
\varepsilon & \varepsilon \varepsilon e & \varepsilon
\end{array}\right) .
\end{aligned}
$$

Die Stellgröße $u^{(i, l)}$ beschreibt den Zeitstempel für die Schalttransition $\lambda_{\text {in }}$ als Eingangssignal von $p^{(i, l)}$. $A_{0}$ und $A_{1}$ beschreiben die Umschaltzeiten des Transitionsvektors $\left(x^{(i, l)}\right)$ in Abhängigkeit der Anfangsbelegung der Nachrichten auf den Plätzen. $B_{0}$ beschreibt die Zeitverzögerung zum Schalten der Eingangstransition $\left(u^{(i, l)}\right)$ in Abhängigkeit der Anfangsbelegung auf den Plätzen. Für die Berechnung der expliziten Bildungsvorschrift wird die nachstehende Form genutzt [CGQ99]

$$
x^{(i, l)}(k+1)=\left(A_{0}^{3} \oplus A_{0}^{2} \oplus A_{0} \oplus E\right)\left(A_{1} x^{(i, l)}(k) \oplus B u^{(i, l)}(k+1)\right),
$$

mit $A^{*}=A_{0}^{3} \oplus A_{0}^{2} \oplus A_{0} \oplus E . A_{0}^{4}=N$, bedeutet, dass $A_{0}$ keine Kreiseigenschaften hat. Die Matrizen $N$ und $E$ werden folgendermaßen beschrieben

$$
N=\left(\begin{array}{cccc}
\varepsilon & \varepsilon & \ldots & \varepsilon \\
\varepsilon & \varepsilon & \ldots & \varepsilon \\
\varepsilon & \varepsilon & \ddots & \vdots \\
\varepsilon & \ldots & \varepsilon & \varepsilon
\end{array}\right) \quad E=\left(\begin{array}{cccc}
e & \varepsilon & \ldots & \varepsilon \\
\varepsilon & e & \ldots & \varepsilon \\
\varepsilon & \varepsilon & \ddots & \vdots \\
\varepsilon & \ldots & \varepsilon & e
\end{array}\right) .
$$

Zusätzlich wird definiert, dass $A^{*} A_{1}=\bar{A}$ und $A^{*} B=\bar{B}$. Das Modell aus Gl.4 ist die Voraussetzung für die Regelung. Hier wird ein modellprädiktiver Ansatz $M P C$ für die Regelung gewählt. Im nächsten Kapitel wird die Vorgehensweise genauer beschrieben.

\section{Modellierung eines dezentralen Regleralgorithmusses}

Die nachstehende Abbildung zeigt das Grundschema der dezentralen Regelung auf. An dieser Stelle soll hervorgehoben werden, dass dezentral bedeutet jedes $S$ im $W C S$ hat seine eigene $M P C$ Reglereinheit. Für ein $M P C$ basiertes automatisches Koexistenzmanagement ist das Prädiktionssignal die Transmission time $\tilde{y}$. Anhand dieses Signals liegen alle Informationen für eine Koexistenzbewertung vor: wenn es eine zusätzliche Zeitverzögerung des Signals gibt oder diese verloren gegangen ist. Für die Regelung werden zwei Bereiche der Nachrichtenübertragung unterschieden. Dafür wird eine Führungsgröße $w$ vorgegeben. Bereich 1: 


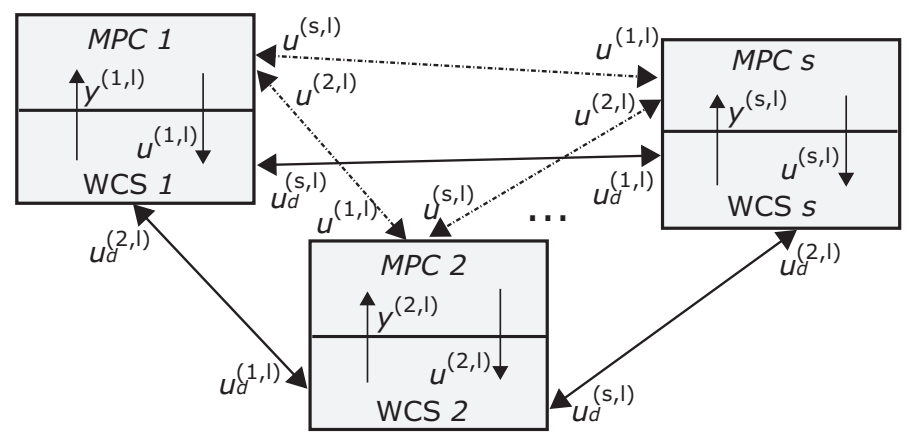

Abb. 4. Struktur der dezentralen Regelung nach [SZ18a]

wenn die Regelgröße $\left(y^{(i, l)} \vee \tilde{y}^{(i, l)}\right)+\triangle \leq w^{(i, l)}$ ist, liegt eine stabile Nachrichtenübertragung vor. Bereich 2: wenn $\left(y^{(i, l)} \vee \tilde{y}^{(i, l)}\right)+\triangle>w^{(i, l)}$ liegt eine instabile Nachrichtenübertragung vor. Die Systemstreuungen werden mit $\triangle$ beschrieben, wobei die Führungsgröße $w$ mit dem Einfluss von $\triangle$ vorgegeben wird. Da es sich bei der MPC -Methodik um ein Optimierungsproblem handelt, wird eine Zielfunktion mit entsprechenden Nebenbedingungen gewählt. Für die Zielfunktion $J$ gilt $J=\sum_{k=k_{1}}^{k_{1}+N_{p}} \max \left(\tilde{y}^{(i, l)}(k)-w^{(i, l)}(k), 0\right)$ mit $k \in\left[k_{1}, k_{1}+N_{p}\right]$, wobei $N_{p}$ der Prädiktionshorizont ist. Das Besondere hierbei ist, dass der Regler nur aktiv wird, wenn Bereich 2 vorliegt. Es wird lediglich eine Stellgröße auf die Prädiktion berechnet und im Anschluss das erste Glied der Folge auf den Prozess implementiert. Daher spielt bei der Definition von $J$ hier nur $\tilde{y}$ eine wesentlich Rolle. An dieser Stelle sei noch einmal erwähnt, dass $w$ hier robust gegenüber des Systemstreuungen gewählt wird. In Kap. 5 wird darauf noch einmal kurz eingegangen. Weiterhin spielt die Stellgröße $u$ keine Rolle in der Formulierung der Zielfunktion. Die Optimierung bezüglich der Energie wird hier noch nicht betrachtet. Das Optimierungsproblem lautet wie folgt [SZ18a]

$$
\min _{u^{(i, l) *}} J,
$$

unter den Nebenbedingungen

$$
\begin{aligned}
\tilde{y}^{(i, l)}(k) & =H \tilde{u}^{(i, l)}(k) \oplus g x^{(i, l)}\left(k_{0}\right) \\
\tilde{y}\left(k_{0}\right) & =y\left(k_{0}\right) \\
\underline{u}^{(j, l)}(k) & \leq \tilde{u}^{(i, l)}(k) \\
u_{\text {sys }}^{(i, l)} & \leq \tilde{u}^{(i, l)}(k) \\
x_{4}^{(i, l)}\left(k_{1}+N_{p}\right) & \leq w^{(i, l)} .
\end{aligned}
$$


$H$ ist die Prädiktionsmatrix und $g$ ist der Vektor für den Einfluss der Anfangsbedingungen $x^{(i, l)}\left(k_{0}\right)$ mit [SHB02] werden $H$ und $g$ folgendermaßen definiert.

$$
\begin{aligned}
H & =\left(\begin{array}{ccccc}
C \bar{B} & \varepsilon & \varepsilon & \ldots & \varepsilon \\
C \bar{A} \bar{B} & C \bar{B} & \varepsilon & \ldots & \varepsilon \\
C \bar{A}^{2} \bar{B} & C \bar{A} \bar{B} & C \bar{B} & \ldots & \varepsilon \\
\vdots & \vdots & \vdots & \ddots & \vdots \\
C \bar{A}^{N_{p}-1} \bar{B} & C \bar{A}^{N_{p}-2} \bar{B} & \ldots & \ldots & C \bar{B}
\end{array}\right) \\
g & =\left(C \bar{A}, C \bar{A}^{2}, C \bar{A}^{3}, \ldots, C \bar{A}^{N_{p}}\right)^{T},
\end{aligned}
$$

wobei $\tilde{y}^{(i, l)}(k)=\left[\hat{y}(k+1), \hat{y}(k+2), \ldots, \hat{y}\left(k+N_{p}\right)\right]^{T}, \tilde{u}^{(i, l)}(k)=[u(k), u(k+1), u(k+$ $\left.2), \ldots, u\left(k+N_{p}-1\right)\right]^{T}$ und $x^{(i, l)}(k=0)=\left[x_{1}\left(k_{0}\right), x_{2}\left(k_{0}\right), x_{3}\left(k_{0}\right), x_{4}\left(k_{0}\right), x_{c}\left(k_{0}\right)\right]^{T}$ ist. Die Endbedingung lautet $x\left(k=k_{1}+N_{p}\right)=\left[x_{1}\left(k=k_{1}+N_{p}\right), x_{2}(k=\right.$ $\left.\left.k_{1}+N_{p}\right), x_{3}\left(k=k_{1}+N_{p}\right), x_{4}\left(k=k_{1}+N_{p}\right), x_{c}\left(k=k_{1}+N_{p}\right)\right]^{T}$. Der Stellgröße $\tilde{u}$ unterliegt zwei Nebenbedingungen. Zunächst die untere Systemgrenze $u_{\text {sys }}$, welche geräteabhängig ist. Gl. 9 stellt den kooperativen Charakter zwischen den Reglern dar. Diese soll aussagen, dass die Transferintervalle der einzelnen $W C S s$ untereinander nicht gleich gewählt werden. Bezüglich des max -Terms in Gl. 6 und Gl. 7 handelt es sich bei der Formulierung um ein nicht konvexes Optimierungsproblem. Der max -Term erfüllt nicht das Kriterium der Superposition. Für das Erhalten eines konvexen Optimierungsproblems wird eine Umformulierung der Gl. 6 und der Gl. 7 statt finden. Dies soll mit Hilfe der linearen Programmierung (englisch Linear Programming $L P$ ) erfolgen. Das Optimierungsproblem wird in die folgende Form gebracht

$$
\min _{r} F^{T} r \quad \text { sodass }\left\{\begin{array}{l}
Q r \leq b \\
Q r=b \\
\underline{r} \leq r \leq \bar{r}
\end{array} .\right.
$$

Es wird eine lineare Zielfunktion $F$ mit linearen Nebenbedingungen in $Q$ und $b$ formuliert. Die Entscheidungsvariable ist $r$ mit Lösungsvektor $r_{\text {solution }}=$ $(\tilde{u}, \tilde{y}, z)$, wobei $z$ eine Pseudovaribale ist. Mit $z$ soll eine Oder -Verknüpfung der nicht linearen Ausdrücke beschrieben werden. Die Variable $z$ muss $\geq \tilde{y}^{(i, l)}(k)-$ $w^{(i, l)}(k)$ oder $\geq 0$ mit Bezug auf die Zielfunktion sein. Mit diesem Sachverhalt wird die ursprüngliche Zielfunktion aus Gl. 6 als

$$
\min _{z^{(i, l) *}} J_{1}=\min _{z^{(i, l) *}} \sum_{k=k_{1}}^{k_{1}+N_{p}} z^{(i, l)}(k), \quad k \in\left[k_{1}, k_{1}+N_{p}\right],
$$

sodass

$$
\begin{aligned}
\tilde{y}^{(i, l)}(k)-z^{(i, l)}(k) & \leq w^{(i, l)}(k) \\
-z^{(i, l)}(k) & \leq 0
\end{aligned}
$$


umformuliert. Dies ermöglicht einen linearen Ausdruck. Für die Umformulierung von Gl. 7 gelten die nachstehenden Schritte

$$
\min _{\tilde{y}^{(i, l) *}} J_{2}=\min _{\tilde{y}^{(i, l) *}} \sum_{k=k_{1}}^{k_{1}+N_{p}} \tilde{y}^{(i, l)}(k), \quad k \in\left[k_{1}, k_{1}+N_{p}\right]
$$

sodass

$$
\begin{aligned}
-\tilde{y}^{(i, l)}(k) & \leq-\bar{C} \bar{A}^{k}(1)-y\left(k_{0}\right)^{(i, l)}(1) \\
-\tilde{y}^{(i, l)}(k) & \leq-\bar{C} \bar{A}^{k}(2)-y\left(k_{0}\right)^{(i, l)}(2) \\
-\tilde{y}^{(i, l)}(k) & \leq-\bar{C} \bar{A}^{k}(3)-y\left(k_{0}\right)^{(i, l)}(3) \\
-\tilde{y}^{(i, l)}(k) & \leq-\bar{C} \bar{A}^{k}(4)-y\left(k_{0}\right)^{(i, l)}(4) \\
-\tilde{y}^{(i, l)}(k) & \leq-\bar{C} \bar{A}^{k}(5)-y\left(k_{0}\right)^{(i, l)}(5) \\
\tilde{u}^{(i, l)}(k)-\tilde{y}^{(i, l)}(k) & \leq-\bar{C}(1)-\bar{B}(1) \\
\tilde{u}^{(i, l)}(k)-\tilde{y}^{(i, l)}(k) & \leq-\bar{C}(2)-\bar{B}(2) \\
\tilde{u}^{(i, l)}(k)-\tilde{y}^{(i, l)}(k) & \leq-\bar{C}(3)-\bar{B}(3) \\
\tilde{u}^{(i, l)}(k)-\tilde{y}^{(i, l)}(k) & \leq-\bar{C}(4)-\bar{B}(4) \\
\tilde{u}^{(i, l)}(k)-\tilde{y}^{(i, l)}(k) & \leq-\bar{C}(5)-\bar{B}(5) \\
\tilde{u}^{(i, l)}(k)-\tilde{y}^{(i, l)}(k) & \leq-\bar{C}(1)-\bar{A}^{(1)} \bar{B}(1) \\
\tilde{u}^{(i, l)}(k)-\tilde{y}^{(i, l)}(k) & \leq-\bar{C}(2)-\bar{A}^{(1)} \bar{B}(2) \\
\tilde{u}^{(i, l)}(k)-\tilde{y}^{(i, l)}(k) & \leq-\bar{C}(3)-\bar{A}^{(1)} \bar{B}(3) \\
\tilde{u}^{(i, l)}(k)-\tilde{y}^{(i, l)}(k) & \leq-\bar{C}(4)-\bar{A}^{(1)} \bar{B}(4) \\
\tilde{u}^{(i, l)}(k)-\tilde{y}^{(i, l)}(k) & \leq-\bar{C}(5)-\bar{A}^{(1)} \bar{B}(5) \\
&
\end{aligned}
$$

wobei $k \in\left[k_{1}, k_{1}+N_{p}\right]$. Die Entscheidungsvariablen befinden sich auf der linken Seite und die Konstanten befinden sich auf der rechten Seite der Ungleichungsnebenbedingungen. Mit der zusätzlichen Umformulierung ist ein konvexes Optimierungsproblem gegeben. Das vollständige Problem lässt sich mit $J=J_{1}+J_{2}$ unter Gl. 16 bis Gl. 38 beschreiben. Wegen der Konvexität wird garantiert, dass die optimale Lösung $r_{\text {solution }}$ für den Koxistenzzustand gefunden wird. 


\section{$5 \quad$ Validierung des Modells für die dezentrale Regelung}

Für die Validierung des Reglers aus Gl. 15 und Gl. 18 sei der nachstehende Hardware-in-the-Loop Aufbau, Abbildung 5, aufgezeigt. Jedes WCS besteht aus

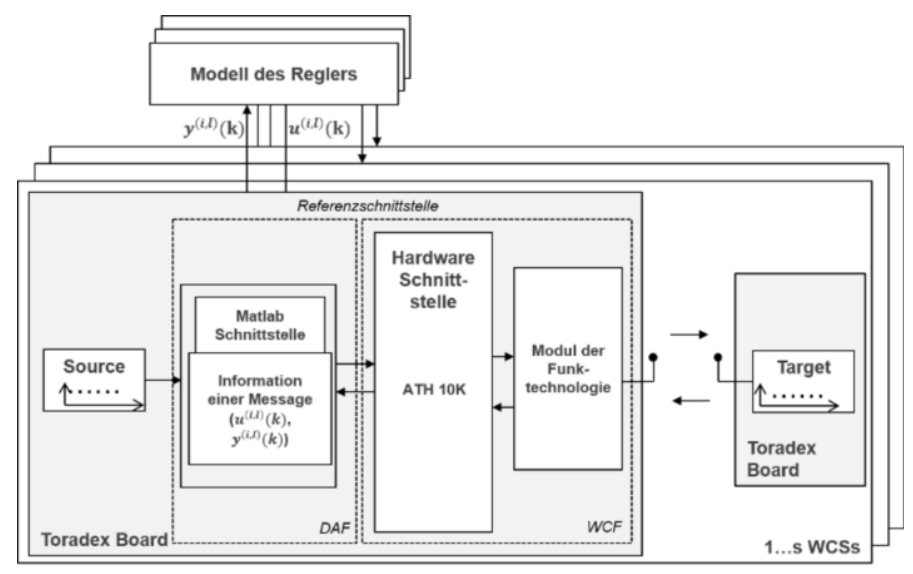

Abb. 5. Beschreibung des Testaufbaus nach [SZ18a]

mindestens zwei identischen Toradex Apalis iMX6 Boards, welche die $S$ und die $T$ darstellen. Weiterhin wird die Analogie von $D A F$ und $W C F$ aus Kap. 3 beschrieben. In Abbildung 5 werden $s W C S s$ aufgezeigt. Jedes $W C S$ hat seinen eigenen Regler, welcher das jeweilige $y$ erhält und das $u$ berechnet. Die Implementierung wurde in Matlab durchgeführt. Die Schnittstelle zwischen Softund Hardware ist mit einem Matlab interface versehen [SZ18b].

Für die Validierung des Reglers wird ein Testszenario im 2, 4 GHz Frequenzband definiert. Dieses Szenario besteht aus zwei $W C S s$ nach dem IEEE802.11n Standard, $p^{(1,1)}$ und $p^{(2,1)}$. Jedes $W C S$ hat die folgende Parametrierung:

Tabelle 1. Parametrierung von $S$ und $T$ für das Test case Szenario [SZJ18]

\begin{tabular}{|c|c|c|}
\hline Parameter & Wert & Einheit \\
\hline Nutzdatenlänge & 1024 & Bit \\
\hline Ausgangsleistung & 10 & $d B m$ \\
\hline Entfernung $S$ zwischen $T$ & 3 & $m$ \\
\hline Führungsgröße $w$ der Transmission time & 450.00 & $\mu s$ \\
\hline
\end{tabular}

Die Führungsgröße $w$ wurde im Vergleich zu [SZJ18] robuster, gegenüber das Streuverhalten, für das störfreie Übertragungsverhalten bestimmt. Beide $W C S s$, 
$p^{(1,1)}$ und $p^{(2,1)}$, fangen zur gleichen Zeit mit ihrer jeweiligen Nachrichtenübertragung an. In Abbildung 6 ist das Einregelverhalten von $p^{(1,1)}$ aufgezeigt. Als

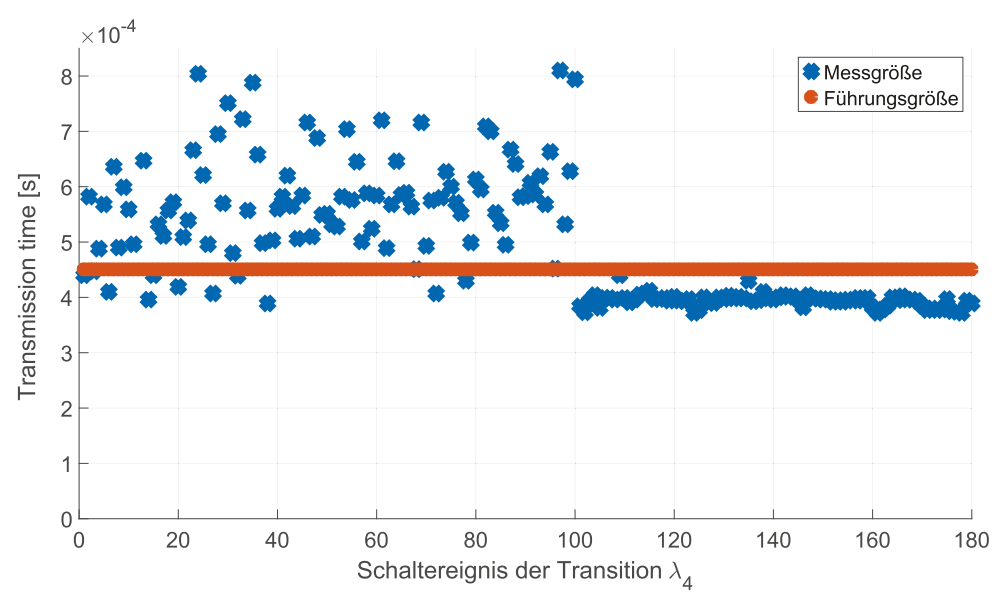

Abb. 6. Validierung des Reglers von $p^{(1,1)}$

Messgröße wird die Transmission time von $p^{(1,1)}$ betrachtet. Bis zu einem Schaltereignis von $\lambda_{4}(k=100)$ sind die Regler von $p^{(1,1)}$ und $p^{(2,1)}$ inaktiv. Hier soll die gegenseitige Beeinflussung demonstriert werden. Ab einem Schaltverhalten von $\lambda_{4}(k=101)$ sind die Regler aktiv. Wie in Abbildung 6 leicht zu sehen ist, wird ein neues Stellsignal so berechnet, dass die Koexistenz vom Regler wieder hergestellt ist.

\section{Schlussfolgerung}

In diesem Beitrag wurde eine dezentrale Regelung für ein automatisches Koexistenzmanagement modelliert und analysiert. Als Algorithmus für den Regler wurde dazu ein modellprädiktives Verfahren mit der max-plus Algebra in Petrinetznotation gewählt. Wegen der nicht konvexen Eigenschaft des Optimierungsproblems wurde eine Umformulierung mit Hilfe der linearen Programmierung vorgenommen. Mit einem realen Testaufbau konnten erste positive Validierungsergebnisse nachgewiesen werden.

\section{Literatur}

[AMW12] Ahmad, K.; Meier, U.; Witte, S.: Predictive Oppertunistic Spectrum Access Using Markov Models. In: 17th IEEE Conference on Emerging Technologies and Factory Automation (ETFA 2012). Krakow, Poland, September 17th 21st 2012. 
[ASM11] Ahmad, K.; Shrestha, G.; Meier, U.: Real-Time Issues of Predictive Modeling for Industrial Cognitive Radios. In: IEEE 9th International Conference on Industrial Informatics - INDIN 2011. Lisbon, Portugal, Juli 26th - 29th 2011.

[CGQ99] Cohen, G.; Gaubert, S.; Quadrat, J.-P.: Max-plus Algebra and System Theory : Where We Are and Where to go now. In: IFAC Annual Reviews in Control. Jgg. 23, S. 207-219, 1999.

[GTS08] Geierhofer, S.; Tong, L.; Sadler, B. M.: Cognitive Medium Access: Constraining Interference Based on Experimental Models. IEEE Journal on Selected Areas in Communications, 26(1), 2008.

[Ha05] Haykin, S.: Cognitive Radio: Brain-Empowered Wireless Communication. IEEE Journal on Selected Areas in Communications, 23(2), 2005.

[IE14] IEC62657-2: Ed1: Industrial Communication Networks: Wireless Communication Networks Part 2: Coexistence Management. Bericht, IEC, 2014.

[In13] International electrotechnical vocabulary Part 351: Control technology (IEC 60050-351:2013, 2013.

[KR15] Kraetzig, M.; Rauchhaupt, L.: Koordinierung heterogener industrieller Funkkommunikation durch ein zentrales Koexistenzmanagement. In: Conference proceedings published on USB stick, ISBN 978-3-944722-33-7. Magdeburg, November 17th - 18th 2015.

[Sc17] Schulze, D.; Rauchhaupt, L.; Kraetzig, M.; Jumar, U.: Coexistence Plant Model for an Automated Coexistence Management. In: 20th IFAC World Congress (WC'17), IFAC-PapersOnLine. Jgg. 50, Toulouse, France, S. 355362 , July 9 th - 14th 2017.

[SHB02] Schutter, B.D.; Heemels, W.; Bemporad, A.: Max-Plus Algebraic Problems and the Extended Linear Complementary Problem - Algorithmic Aspects. In: 15th IFAC World Congress. IFAC, Barcelona, Spain, S. $151-156$, July 21 th - 26th 2002.

[SR16] Schulze, D.; Rauchhaupt, L.: A Control Engineering Approach for an Automated Coexistence Management. In: 4th IFAC Symposium on Telematics Application (TA'16), IFAC-PapersOnLine. Jgg. 49, Porto Alegre, Brazil, S. $284-289$, November 6th - 9th 2016.

[SRJ17] Schulze, D.; Rauchhaupt, L.; Jumar, U.: Coexistence for Industrial Wireless Communication Systems in the Context of Industrie 4.0. In: Australian and New Zealand Control Conference (ANZCC'17). Gold Coast, Australia, December 17th - 20th 2017.

[Su12] Sun, C.; Villardi, G. P.; Lan, Z.; Alemseged, Y. D.; Tran, H. N.; Harada, H.: Optimizing the Coexistence Performance of Secondary-User Networks Under Primary-User Constraints for Dynamic Spectrum Access. IEEE Transactions on Vehicular Technology, 61(8):3665 - 3676, 2012.

[SZ18a] Schulze, D.; Zipper, H.: A Decentralised Control Algorithm for an Automated Coexistence Management. In: 2018 IEEE Conference on Decision and Control (CDC'18). Miami Beach, USA, December 17th-19th, angenommen 2018.

[SZ18b] Schulze, D.; Zipper, H.: Koexistenz drahtloser Kommunikationssysteme im Kontext von Industrie 4.0. In: AUTOMATION 2018, Seamless Convergence of Automation \& IT. VDI Verlag GmbH, Baden-Baden, Germany, July 3th - 4th 2018.

[SZJ18] Schulze, D.; Zipper, H.; Jumar, U.: Modelling and Simulation of the Interference Behaviour in Industrial Wireless Communication. In: IEEE International Conference on Industrial Cyber-Physical Systems (ICPS-2018). Saint Petersburg, Russia, May 15th - 18th 2018. 
[YA09] Yuecek, F.; Arslan, H.: A Survey of Spectrum Sensning Algorithm for Cognitive Radio Applications. Communication Surveys and Tutorials IEEE, 11(1):116-130, 2009.

Open Access Dieses Kapitel wird unter der Creative Commons Namensnennung 4.0 International Lizenz (http://creativecommons.org/licenses/by/4.0/deed.de) veröffentlicht, welche die Nutzung, Vervielfältigung, Bearbeitung, Verbreitung und Wiedergabe in jeglichem Medium und Format erlaubt, sofern Sie den/die ursprünglichen Autor(en) und die Quelle ordnungsgemäß nennen, einen Link zur Creative Commons Lizenz beifügen und angeben, ob Änderungen vorgenommen wurden.

Die in diesem Kapitel enthaltenen Bilder und sonstiges Drittmaterial unterliegen ebenfalls der genannten Creative Commons Lizenz, sofern sich aus der Abbildungslegende nichts anderes ergibt. Sofern das betreffende Material nicht unter der genannten Creative Commons Lizenz steht und die betreffende Handlung nicht nach gesetzlichen Vorschriften erlaubt ist, ist für die oben aufgeführten Weiterverwendungen des Materials die Einwilligung des jeweiligen Rechteinhabers einzuholen. 